\title{
PROPERNESS OF LIE ALGEBRAS AND ENVELOPING ALGEBRAS. I
}

\author{
WALTER MICHAELIS
}

\begin{abstract}
An associative unitary (respectively, Lie) algebra is said to be proper in case the intersection of all of its cofinite two-sided (respectively, Lie) ideals is zero. Using the Hopf algebra structure of $U L$, it is shown that over a field of characteristic zero a Lie algebra is proper if and only if its universal enveloping algebra is proper. (In the finite-dimensional case this provides a new proof of a result of Harish-Chandra.) The analogous result for Lie $p$ algebras and their restricted universal enveloping algebras holds and is proved by the same technique.
\end{abstract}

Notational conventions. Throughout this paper, $p$ will denote a prime number. Barring mention to the contrary, we work over an arbitrary ground field $K$ except that when Lie $p$-algebras (=restricted Lie algebras of characteristic $p$ ) are discussed, the ground field must have characteristic $p$ (cf. $[1$, p. 78 , Exercise 20] or [7, p. 187, Definition 4]). The unmodified words "algebra" and "coalgebra" shall, respectively, mean "associative unitary algebra" and "associative counitary coalgebra." If $A$ is an algebra, $\mathcal{L}(A)$ shall denote the Lie algebra whose underlying vector space coincides with that of $A$, and whose bracket is defined for all elements $x$ and $y$ of $A$ by $[x, y]_{\mathcal{L}(A)}=x \cdot y-y \cdot x$ where "." denotes the multiplication of $A$; while $\mathcal{L}_{p}(A)$ shall denote the Lie $p$-algebra whose underlying Lie algebra is $\mathcal{L}(A)$ and whose $p$-mapping, $x \mapsto x^{[p]}$, is the $p$ th power mapping, $x \mapsto x^{p}$, of the algebra $A$. For any algebra map $f: A \rightarrow B, \mathcal{L}(f): \mathcal{L}(A) \rightarrow \mathcal{L}(B)$ (respectively, $\mathcal{L}_{p}(F): \mathcal{L}_{p}(A) \rightarrow$ $\mathcal{L}_{p}(B)$ ) shall denote the map of Lie algebras (respectively, Lie $p$-algebras) which coincides with $f$ as a vector space map. For any Lie algebra $L, U L$ shall denote the universal enveloping algebra of $L$; while for any Lie $p$-algebra $L, V L$ shall denote the restricted universal enveloping algebra of $L$; furthermore, $i$ and $j$ shall denote, respectively, the canonical injections $i: L \rightarrow \mathcal{L}(U L)$ and $j: L \rightarrow \mathcal{L}_{p}(V L)$. Occasionally, the vector space whose only element is zero shall be denoted by 0 , and, occasionally, the end of proof shall be landmarked by the symbol

DEFINITION 1. (a) If $A$ is an algebra (respectively, a Lie algebra), denote by C.I. $(A)$ the collection of all cofinite two-sided ideals (respectively, all cofinite Lie

Received by the editors December 26, 1979 and, in revised form, August 1, 1980 and June 3, 1986. The author spoke on various stages of development of this paper at Annual Meetings of the AMS on the following dates: January 6, 1980 (Abstract \#773-16-13), January 8, 1981 (Abstract \#783-16-7), January 27, 1984 (Abstract \#809-16-164), January 9, 1986 (Abstract \#825-16-363).

1980 Mathematics Subject Classification (1985 Revision). Primary 17B35; Secondary 16A24, $17 \mathrm{~B} 10$.

The author gratefully acknowledges partial support from a SUNY at Buffalo Individual Faculty Development Grant during the preparation of this paper. 
ideals) of $A$, and set

$$
R(A)=\bigcap_{I \in \text { C.I. }(A)} I
$$

(b) If $A$ is a Lie $p$-algebra, denote by C.p-I.(A) the collection of all cofinite Lie $p$-ideals of $A$, and set

$$
R_{p}(A)=\bigcap_{I \in \text { C.p-I. }(A)} I .
$$

DEFinition 2. (a) An algebra (respectively, a Lie algebra) $A$ is said to be proper (or residually finite) in case $R(A)=0$.

(b) A Lie $p$-algebra $A$ is said to be $p$-proper in case $R_{p}(A)=0$.

The aim of the present paper is to establish the following:

MAIN THEOREM 3. (a) Over a field of characteristic 0, a Lie algebra is proper if and only if its universal enveloping algebra is proper, i.e.,

$$
R(L)=0 \Leftrightarrow R(U L)=0 .
$$

(b) Over a field of characteristic p, a Lie p-algebra is p-proper if and only if its restricted universal enveloping algebra is proper, i.e.,

$$
R_{p}(L)=0 \Leftrightarrow R(V L)=0 .
$$

In [11], we show that Theorem 3(a) is also true over fields of characteristic $p$.

The proof of Theorem 3 is Hopf-theoretic, so we pause first to introduce the requisite terminology. In what follows, we use the term "Hopf algebra" to refer to what Sweedler [13] calls a "bialgebra." In this regard, our terminology parallels that of Milnor and Moore [12].

DEFINITION 4. (a) If $H$ is a Hopf algebra with diagonal $\Delta: H \rightarrow H \otimes H$, then $P(H)$, the space of primitives of $H$, is defined by setting

$$
P(H)=\{z \in H \mid \Delta z=z \otimes 1+1 \otimes z\}
$$

where 1 is the identity element of $H$.

(b) A Hopf algebra $H$ is said to be primitively generated in case $P(H)$ generates $H$ as an algebra.

(c) A subspace $I$ of a Hopf algebra $H$ is a coideal in case $\Delta(I) \subseteq I \otimes H+H \otimes I$ and $I \subseteq$ ker $\varepsilon$, where $\varepsilon: H \rightarrow K$ is the counit of $H$.

Recall that $U L$ (respectively, $V L$ ) has a Hopf algebra structure for which the elements of $i L$ (respectively, $j L$ ) are primitive (cf. [1, p. 115, Proposition 7 and, respectively, pp. 180-181, Exercise 12.a]).

To establish Theorem 3, we make use of six facts which we state next as theorems. Of these, the first two are new (as far as we know) while the last four are well known. Proofs of the new results will follow the proof of Theorem 3.

THEOREM 5. For any Hopf algebra $H, R(H)$ is a coideal of $H$.

THEOREM 6. (a) If $L$ is a Lie algebra over a field (of any characteristic), then $R(U L) \cap i L=i[R(L)]=R(i L)$.

(b) If $L$ is a Lie p-algebra, then $R(V L) \cap j L=j\left[R_{p}(L)\right]=R_{p}(j L)$. 
THEOREM 7. (a) UL is a primitively generated Hopf algebra.

(b) $V L$ is a primitively generated Hopf algebra.

Theorem 7 is an immediate consequence of the fact that $i L$ (respectively, $j L$ ) generates $U L$ (respectively, $V L$ ) as an algebra.

THEOREM 8. (a) In characteristic zero, $i L=P(U L)$.

(b) In characteristic $p, j L=P(V L)$.

Theorem $8(\mathrm{a})$ is known as Friedrichs' Theorem (cf. [4, p. 19, Footnote 3; 7, p. 170, Theorem 9]). For proofs of $8(\mathrm{a})$ and (b) see [12, Theorems 5.18.1 and 6.11.1]

THEOREM 9. If $I$ is a coideal of a primitively generated Hopf algebra $H$, then

$$
I \cap P(H)=0 \quad \Rightarrow \quad I=0 .
$$

Theorem 9 is an immediate consequence of Corollary 11.0.2, Exercise 2, p. 224, and Theorem 9.2.2 in Sweedler [13].

THEOREM 10. (a) $i: L \rightarrow \mathcal{L}(U L)$ is injective.

(b) $j: L \rightarrow \mathcal{L}_{p}(V L)$ is injective.

Proofs of 10(a) and (b) can be found in [7, p. 160, Corollary 1 and, respectively, p. 191, Theorem 12].

With the results of Theorems 5-10 at hand, we now establish the Main Theorem.

PROOF OF THEOREM 3. For part (a), notice that if $R(U L)=0$, then

$$
i[R(L)]=R(U L) \cap i L=0,
$$

so $R(L)=0$ because $i$ is injective. Conversely, if $R(L)=0$, then

$$
R(U L) \cap P(U L)=R(U L) \cap i L=i[R(L)]=i(0)=0,
$$

and thus $R(U L)=0$ because $R(U L)$ is a coideal of the primitively generated Hopf algebra $U L$. This, modulo proofs of Theorems 5 and 6 , given below, completes the proof of (a). The proof of (b) is exactly analogous to that of (a). (N.B.: In characteristic $p$,

$$
P(U L)=\operatorname{Span}\left\{(i x)^{p^{k}} \mid x \in L \text { and } k \in \mathrm{N}\right\}
$$

where $\mathrm{N}$ is the set of natural numbers (cf. [2, pp. 3-14, Corollaire]), so $i L \varsubsetneqq P(U L)$. Consequently, the proof just given to establish (a) in characteristic zero does not work in characteristic $p$. For details of what to do in that case see [11].)

For the proof of Theorem 5 we require the following results from the theory of coalgebras and Hopf algebras.

DEFINITION 11. If $V$ is a vector space over an arbitrary ground field, if $I$ is a vector subspace of $V$, and if $J$ is a vector subspace of $V^{*}$, then $I^{\perp}$, the annihilator of $I$ in $V^{*}$, is defined by

$$
I^{\perp}=\left\{f \in V^{*} \mid I \subseteq \operatorname{ker} f\right\},
$$

while $J^{\Perp}$, the annihilator of $J$ in $V$, is defined by

$$
J^{\Perp}=\{v \in V \mid f(v)=0 \text { for all } f \in J\} .
$$

PROPOSITION 12. Given a coalgebra $(C, \Delta, \varepsilon)$ with comultiplication $\Delta: C \rightarrow$ $C \otimes C$ and counit $\varepsilon: C \rightarrow K$, let $\lambda: K \rightarrow K^{*}$ be the left regular representation 
of the ground field $K$ on itself (defined by $\lambda(k)\left(k^{\prime}\right)=k \cdot k^{\prime}$ for each $k, k^{\prime} \in K$ ), and let $\rho: H^{*} \otimes H^{*} \rightarrow(H \otimes H)^{*}$ be the natural vector space injection defined for elements $f$ and $g$ of $H^{*}$ by $\rho(f \otimes g)=\varphi_{K} \circ(f \otimes g)$ where $\varphi_{K}: K \otimes K \rightarrow K$ is the multiplication of the ground field $K$. Then the following results hold:

(a) $\left(C^{*}, \Delta^{*} \circ \rho, \varepsilon^{*} \circ \lambda\right)$ is an algebra, called the dual algebra of $C$.

(b) If $A$ is a subalgebra of $C^{*}$, the dual algebra of $C$, then $A^{\Perp}$ is a coideal of $C$.

For the proof of Proposition $12(\mathrm{a})$ and (b) the reader is referred to $[13$, p. 9 , Proposition 1.1.1 and p. 19, Proposition 1.4.6.b].

PROPOSITION 13. If $H$ is a Hopf algebra, iet

$$
H^{0}=\bigcup_{I \in C . I .(H)} I^{\perp}=\left\{f \in H^{*} \mid \text { ker } f \supseteq I \text { for some } I \in C . I .(H)\right\} .
$$

Then the following results hold:

(a) $H^{0}=\sum_{I \in C . I .(H)} I^{\perp}$, and

(b) $H^{0}$ is a subalgebra of the dual algebra $H^{*}$ of the (underlying) coalgebra (of) $H$.

ProOF. (a) Clearly $\bigcup I^{\perp} \subseteq \sum I^{\perp}$. The reverse inclusion is an immediate consequence of the following two observations.

(1) $\left(I_{1}^{\perp}+I_{2}^{\perp}+\cdots+I_{n}^{\perp}\right)=\left(I_{1} \cap I_{2} \cap \cdots \cap I_{n}\right)^{\perp}$. (This is Proposition A.5.b of Sweedler $[13$, p. 320].)

(2) The intersection of two (and hence of finitely many) cofinite subspaces of a vector space is again cofinite. (This is Proposition A.6 of Sweedler [13, p. 321].)

(b) For a proof, see [13, p. 110, Lemma 6.0.1.c and pp. 122-123, §6.2].

PROOF OF THEOREM 5.

$$
\left(H^{0}\right)^{\Perp}=\left[\sum_{I \in \text { C.I. }(H)} I^{\perp}\right]^{\Perp}=\bigcap_{I \in \text { C.I. }(H)} I^{\perp \Perp}=\bigcap_{I \in \text { C.I. }(H)} I=R(H) .
$$

Theorem 6 belongs to the province of representation theory. For its proof we make use of part (b) of the following two lemmas (of which part (c) of Lemma 14 is included for the sole purpose of relating Theorem 3 to a result of Harish-Chandra (cf. Remark 16, below)).

LEMMA 14. Let $A$ be an algebra (respectively, a Lie algebra). Then:

(a) The cofinite ideals of $A$ (two-sided or Lie-as the case may be) are precisely the kernels of finite-dimensional representations of $A$.

(b) $x \in R(A) \Leftrightarrow f(x)=0$ for every finite-dimensional representation $f$ of $A$.

(c) $R(A)=0 \Leftrightarrow$ for each nonzero element $z \in A$, there exists a finite-dimensional representation $f$ of $A$ for which $f(z) \neq 0$, i.e., $A$ has sufficiently many representations to separate points.

PROOF. The nontrivial implication of (a) is an easy consequence of the fact that every finite-dimensional algebra has a faithful finite-dimensional representation: In the case of an associative unitary algebra $A$, the left (or right) regular representation of $A$ on itself will work. In the case of a Lie algebra $L$, this is the content of the Ado-Iwasawa Theorem (cf. [7, pp. 202 and 204]. Clearly, (b) follows at once from (a), while (c) follows readily from (b). 
For the next lemma, we recall that if $A$ is a Lie $p$-algebra, then a finitedimensional $p$-representation (=restricted representation) of $A$ is a morphism $f: A \rightarrow \mathcal{L}_{p}($ End $V)$ of Lie $p$-algebras from $A$ into the Lie $p$-algebra of endomorphisms of a finite-dimensional vector space $V$.

LEMMA 15. Let $A$ be a Lie p-algebra. Then:

(a) The cofinite Lie $p$-ideals of $A$ are precisely the kernels of finite-dimensional $p$-representations of $A$.

(b) $x \in R_{p}(A) \Leftrightarrow f(x)=0$ for every finite-dimensional p-representation $f$ of $A$.

The proof of Lemma 15 is exactly analogous to that of Lemma 14 because every finite-dimensional Lie $p$-algebra has a faithful finite-dimensional $p$-representation (cf. [7, p. 191, Theorem 12]).

REMARK 16. Since finite-dimensional Lie algebras clearly are proper, our Main Theorem (Theorem 3) generalizes the result of Harish-Chandra (cf. [5, p. 905, Theorem 1] or [3, p. 84, Theorem 2.5.7]) which asserts that over a field of characteristic zero, the universal enveloping algebra of a finite-dimensional Lie algebra has "sufficiently many representations." Our proof is of interest even in this case because it is short, noncomputational, and Hopf-theoretic. Note: For a finitedimensional Lie algebra $L$, Theorem 6 (a) just says $R(U L) \cap i L=0$, and this fact is an immediate consequence of the Ado-Iwasawa Theorem (which asserts that for any finite-dimensional Lie algebra $L$ there is a cofinite two-sided ideal $I$ of $U L$ such that $I \cap i L=0)$.

Proof OF THEOREM 6(a). For convenience, let F.D.R. $(A)$ denote the set of all finite-dimensional representations of $A$. To establish the inclusion $i[R(L)] \subseteq$ $\mathcal{R}(U L) \cap i L$, let $x \in R(L)$ and let $G \in$ F.D.R. $(U L)$ be arbitrary. Then $\mathcal{L}(G) \circ i \in$ F.D.R. $(L)$ and so (by Lemma 14(b))

$$
x \in R(L) \Rightarrow 0=\mathcal{L}(G) \circ i(x)=G(i x) .
$$

Accordingly, $i x \in R(U L)$. This proves that $i: L \rightarrow \mathcal{L}(U L)$ carries the subspace $R(L)$ of $L$ into the subspace $R(U L)$ of $\mathcal{L}(U L)$, and consequently that

$$
i[R(L)] \subseteq R(U L) \cap i L .
$$

For the reverse inclusion, suppose that $i x \in R(U L) \cap i L$, and let $f \in$ F.D.R. $(L)$ be arbitrary. By the universal mapping property satisfied by $(U L, i)$, there exists an $F \in$ F.D.R. $(U L)$ such that $f=\mathcal{L}(F) \circ i$. Then

$$
i x \in R(U L) \Rightarrow 0=F(i x)=\mathcal{L}(F) \circ i(x)=f(x)
$$

and consequently, by Lemma $14(\mathrm{~b}), x \in R(L)$; thus $i x \in i[R(L)]$, thereby establishing the inclusion

$$
R(U L) \cap i L \subseteq i[R(L)] .
$$

[N.B.: In establishing the inclusion $R(U L) \cap i L \subseteq i[R(L)]$, we used the nontrivial implication (" $\Leftarrow$ ") from Lemma 14(b), and that required the Ado-Iwasawa Theorem.]

The equality $i[R(L)]=R(i L)$ follows at once from the following more general result: If $f: A \rightarrow B$ is an algebra (or Lie algebra) isomorphism, then $f[R(A)]=$ $R[f(A)]$.

The proof of Theorem 6(b) is completely analogous to that of Theorem 6(a). 
REMARK. An alternate way of formulating Theorem 6(a) is to assert that there is a commutative diagram

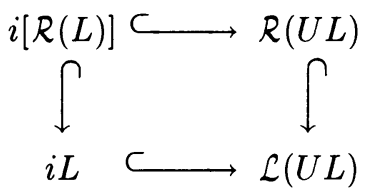

whose morphisms are inclusions, and that this diagram is a pull-back in the category of Lie algebras. (A similar comment holds in connection with Theorem 6(b).)

REMARK 17. In [5, pp. 908-909, Proposition 1 and the comment which follows], Harish-Chandra observes that his result [5, p. 905, Theorem 1] (that $U L$ has sufficiently many representations) is a generalization of Ado's Theorem. The next proposition strengthens this result and places it in an appropriate context.

Proposition 18. Let $A$ be a proper algebra (associative or Lie). Then for any given finite-dimensional subspace $V$ of $A$, there exists an $I \in C . I .(A)$, depending on $V$, such that $V \cap I=0$. (The analogous result holds for p-proper Lie p-algebras.)

Proof. Choose $I \in$ C.I. $(A)$ so that $\operatorname{dim}(V \cap I)$ is minimal. Because C.I. $(A)$ is closed under finite intersections, $V \cap I=V \cap I \cap J \subseteq J$ for all $J \in$ C.I. $(A)$. Thus, $V \cap I \subseteq R(A)=0$.

REMARK 19. (a) It is interesting to note that the statement " $U L$ is proper" is just a statement about the algebra structure of $U L$. Our proof, however, makes use of the additional, underlying coalgebra structure of $U L$.

(b) To establish the properness of $U L$ from that of $L$, we used the Ado-Iwasawa Theorem. Conversely, if one knew by some other means that $U L$ were proper-as one does, for instance, in the case of nilpotent Lie algebras (cf. [6, p. 230, Lemma 4.1] - one would obtain an immediate proof of the Ado-Iwasawa Theorem from Proposition 18 upon taking $V=i L$. Thus, for finite-dimensional Lie algebras $L$, the properness of $U L$ is equivalent to the Ado-Iwasawa Theorem.

(c) The properness of $U L$ for finite-dimensional $L$ is also equivalent to a theorem about Lie coalgebras (cf. [9, p. 39, Theorem] or [10, p. 99, Theorem 1.10]).

(d) Given any algebra $A$ (associative or Lie) we may endow $A$ with a topology by taking as a neighborhood basis of the origin the collection C.I. $(A)$ of all cofinite ideals of $A$. Proposition 18 may then be viewed as saying that whenever the resulting topology is Hausdorff, then any finite-dimensional subspace of $A$ is discrete (cf. [8, p. 76, Item (25.b) of Chapter II]). Note: For an associative algebra $A$, the space of all linear functions from $A$ to $K$ which are continuous when $A$ is given the "cofinite-ideal-topology" and $K$ is given the discrete topology is precisely $A^{0}$ (defined as in Proposition 13). For this reason, $A^{0}$ is sometimes called the continuous linear dual of $A$.

ACKNOWLEDGment. I wish to thank Wim H. Schikhof (of Nijmegen, The Netherlands) for helpful discussions.

\section{REFERENCES}

1. N. Bourbaki, Lie groups and Lie algebras, Part I, Chapters 1-3, Addison-Wesley, Reading, Mass., 1975.

2. P. Cartier, Hyperalgèbres et groupes de Lie formels, Séminaire "Sophus Lie," 2e année: 1955/56, Secrétariat Math., Paris, 1957. 
3. J. Dixmier, Enveloping algebras, North-Holland, Amsterdam, New York, and Oxford, 1977.

4. K. O. Friedrichs, Mathematical aspects of the quantum theory of fields, Part V, Comm. Pure Appl. Math. 6 (1953), 1-72.

5. Harish-Chandra, On representations of Lie algebras, Ann. of Math. (2) 50 (1949), 900-915.

6. G. P. Hochschild, Basic theory of algebraic groups and Lie algebras, Springer-Verlag, New York, Heidelberg, and Berlin, 1981.

7. N. Jacobson, Lie algebras, Wiley-Interscience, New York, London, and Sydney, 1962.

8. S. Lefschetz, Algebraic topology, Amer. Math. Soc., Providence, R.I., 1942.

9. W. Michaelis, Lie coalgebras, Adv. in Math. 38 (1980), 1-54.

10. __ The dual Poincaré-Birkhoff-Witt theorem, Adv. in Math. 57 (1985), 93-162.

11. __ Properness of Lie algebras and enveloping algebras, II, Lie Algebras and Related Topics (Proceedings of a Summer Seminar held June 26-July 6, 1984) (D. J. Britten, F. W. Lemire, and R. V. Moody, eds.), Canad. Math. Soc. Conf. Proc., vol. 5, Amer. Math. Soc., Providence, R.I., 1986, pp. 265-280.

12. J. W. Milnor and J. C. Moore, On the structure of Hopf algebras, Ann. of Math. (2) 81 (1965), 211-264.

13. M. E. Sweedler, Hopf algebras, Benjamin, New York, 1969.

Department of Mathematics, University of New Orleans, New Orleans, LOUISIANA 70148 